\title{
REDES, COMPLEJIDAD Y ETNOMUSICOLOGÍA: REGISTRO DE UN CASO AFROCONTEMPORÁNEO
}

\author{
NETWORKS, COMPLEXITY AND ETNOMUSICOLOGY: \\ REGISTER OF AN AFROCONTEMPORARY CASE
}

\section{RESUMEN}

Hasta hace poco la conexión entre análisis de redes sociales y etnomusicología se estableció de manera marginal dentro de ambos campos. El regreso de las redes sociales y su análisis, presenta diferentes desafíos respecto a las maneras mediante las cuales se instauran las relaciones entre actores de diversa índole y sus complejos socioculturales. Este trabajo presenta como resultado la visualización y análisis de las relaciones étnicas y de género de los jóvenes afro raperos de la ciudad de Cali (Colombia) en la década de los años noventa, comprendidas desde la metodología propia del Análisis de Redes Sociales (ARS) a partir del estudio de su acervo musical, lo cual configura un intento de conciliación entre el ARS y la etnomusicología. Se concluye con la discusión de las dinámicas de las redes estudiadas, sus características estructurales y aspectos relevantes a nivel de su comunicación cultural.

Palabras clave: análisis de redes sociales; complejidad; comunicación; etnomusicología; rap.

\section{ABSTRACT}

Until recently the connection between social network analysis and ethnomusicology was marginally established within both fields. The return of social networks and their analysis, presents different challenges regarding the ways in which the relationships between different actors and their sociocultural complexes are established. This study presents as a result the visualization and analysis of the ethnic and gender relations of the young Afro-rappers of the city of Cali (Colombia) in the decade of the nineties, understood from the methodology of social network analysis (ARS) starting from the study of its musical heritage, which constitutes an attempt at conciliation between the ARS and ethnomusicology. It concludes with the discussion of the dynamics of the studied networks, their structural characteristics and relevant aspects at the level of their cultural communication.

Keywords: communication; complexity; ethnomusicology; rap; social network analysis. 


\section{INTRODUCCIÓN}

En su libro "La Nueva Comunicación", Yves Winkin (1976) consideraba que se pueden definir dos modelos de comunicación como mínimo; 1) el modelo telegráfico, y 2) el modelo orquestal. El primero se puede considerar como un aspecto lineal de la comunicación, regido por una estructura codificada el cual operaría con una lógica propia debido a su estructuración.

Por otra parte, el modelo orquestal de la comunicación se presenta como una interacción compleja entre los músicos (o actores) el cual media las relaciones, los encuentros y las participaciones, no como fines en sí mismos, mejor como intercambios comunicativos en los fenómenos sociales.

En esta analogía se hace importante rescatar la idea de la necesidad de profundizar tanto en el habla como en el lenguaje (Winkin 1976:107). Vemos en este punto la emergencia de puntualizar los aspectos que confluyen en las prácticas musicales como la oralidad y la corporalidad en la materialización de objetos culturales, por ejemplo, los discos y grabaciones musicales.

Este marco de trabajo invita a identificar, en el análisis de los problemas derivados de la comunicación en diversos contextos sociales, la concepción de las formas de expresividad mediante las cuales los grupos, redes, comunidades o escenas sociales establecen vínculos más allá de los espacios clásicos de comunicación próxima.

Una perspectiva reciente la cual vincula el análisis de redes sociales y los procesos de comunicación de diversos conglomerados identifica los conceptos de homofilia - en análisis de redes con lazos fuertes de mayor interacción o intensidad (Reynoso 2011) y heterofilia como fundamentos básicos de su descripción. Por homofilia se entiende la tendencia de los individuos a interactuar con otros similares, y sobre la heterofilia se dice que es la propensión de los individuos a relacionarse con otros seres diferentes a ellos.

En su naturaleza la homofilia es mucho más usual que la heterofilia, y presenta cierta cierto rasgo de ubicuidad a nivel evolutivo (Fu, Nowak, Christakis y Fowler 2012). La recurrencia de este fenómeno se basa a nivel biológico sobre la predilección del individuo por otros similar en su fenotipo, a nivel cultural esto se manifiesta en la inclinación de los sujetos por otros pares de filiación etno-racial. En algunos casos inclusive se pueden presentar casos de polarización cultural, lo que equivale a la influencia social que moldea los comportamientos grupales y sus dinámicas de interacción con una única convergencia hacia el endogrupo (grupo propio) cerrando el acceso a cambios o diferentes dinámicas de comunicación grupales (Axelrod 1997).

Para algunos autores, la comunicación interna respecto a "la homofilia puede producir ventajas de aptitud porque las personas que usan el mismo modo de comunicación pueden actuar juntas de manera más efectiva", lo cual puede explicar el poder de un lenguaje cultural como la música y sus expresividades características lo cual "puede ayudar a promover una tendencia general a buscar individuos similares con quienes cooperar o interactuar" (Fu et al. 2012:5).

Es interesante reconocer que estos y otros ejemplos definen la comunicación personal (cara a cara) e interpersonal (indirecta) en un sentido demográfico como posibilidades de ser más fuertes a partir de las preferencias subjetivas o individuales con respecto a la similitud de los posibles compañeros o colegas destinados a interactuar en una red social determinada (Kossinets y Watts 2009).

En la actualidad algunos estudiosos consideran que en las sociedades occidentales uno de los atributos principales del relacionamiento es la conexión con otros seres y grupos sociales (Barabási 2003), lo cual exige reconocer en estas conexiones la compleji- 
dad que subyace a las prácticas sociales más allá de los fenómenos, objetos o procesos bajo estudio, desde una posible ecuanimidad cultural de modo inherente a los procesos investigativos tal como planteaba Lomax (1977). Esta ecuanimidad pretende abordar de manera inter o transdisciplinaria las diversas problemáticas humanas a través de recursos tecnológicos comunicativos en pro de la equidad cultural, la cual consideramos también debe ser investigativa.

A nivel etnomusicológico hay ciertas lógicas que subyacen a los procesos de la oralidad y aunque puedan presentarse como convencionales de manera 'apresurada' para muchos estudiosos, la oralidad presenta inmensas dificultades en la comprensión de las prácticas artísticas y la transmisión de sus objetos producidos (Chemillier 2002).

Respecto a su estatuto epistemológico en las sociedades occidentales la complejidad de la oralidad también precisa desafíos, por ejemplo, en la dificultad de definir sus mecanismos intrínsecos en los procesos de comunicación culturales, no solo desde el sentido común (Ong 2006). Hemos tomado el ejemplo de la oralidad debido a la disposición inicial de la música rap, la cual es en efecto oral, tanto en los contextos latinoamericanos de su desarrollo como en el resto del planeta (Mitchell 2001), fenómenos sociales que además, forman parte del extenso repertorio de las "artes verbales afrodescendientes" (Finnegan 1992).

Dado que la etnomusicología señaló las problemáticas de reconocer los límites entre el discurso hablado, las canciones y los performances, List (1963) manifiesta que dicha distinción depende de los usos culturales de lo musical y lo oral, ya que en algunos casos la música predomina sobre lo hablado en otros se presenta esta relación de manera inversa. Esta diferencia - según List (1963) - es significativa en muchos casos (Finnegan 1992:131) no solo desde una perspectiva etic (punto de vista de investigador), también figura como fenómeno emic (punto de vista de la comunidad) (Headland, Pike y Marvin 1990).

Sobre un nivel de las redes sociales otros investigadores conectaron la idea de la influencia entre artistas, como procesos emergentes y complejos los cuales se los puede considerar redes con claros patrones de asociación evidenciados entre diversas relaciones e influencias de artistas musicales (Cano y Koppenberger 2004).

Este fenómeno también se presenta en las redes de recomendaciones de artistas musicales, algunos ejercicios investigativos han encontrado que existen tipos similares de percepción en el conocimiento musical, contribuye a un conocimiento complejo y quizá profundo de la música y lo musical (Cano, Celma y Koppenberger 2006).

Este campo se conoce como minería de datos musicales y hace relación a las propiedades de la música por ejemplo, desde la teoría de grafos, hasta llegar a los tipos de redes de influencia y recomendación, índices de tipificación musical, fuentes de datos musicales y su procesamiento, información musical, taxonomías musicales, procesos de comunicación musical y las redes basadas en el conocimiento musical, entre otros aspectos (Li, Ogihara y Tzanetakis 2012).

En la investigación representada en la figura 1 , se establece la conexión entre la comunicación y la música en el marco de la cultura y el ARS para instaurar el reconocimiento de las prácticas de relacionamiento social que los liricistas del rap de la ciudad de Cali, auto-reconocidos como afro en un nivel social y cultural, según las perspectivas indagadas en los raperos caleños y su genealogía cultural.

En su mayoría residían en las comunas 13, $14,15,16$ y 21 , que se pueden apreciar en la figura 1 y pertenecían a los estratos socioeconómicos 1, 2 y 3 de la ciudad de Cali en la década del noventa, lo que se demostraba en su organización diferenciada (Luján 


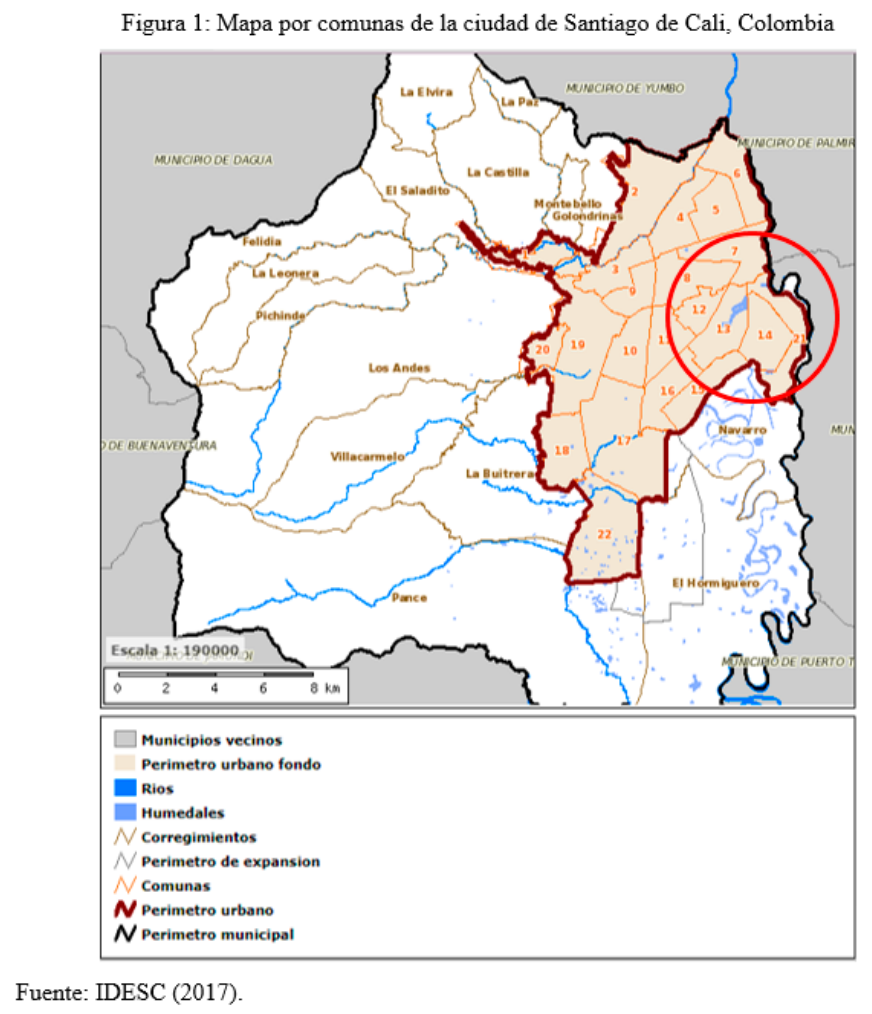

2016). Este territorio parte del conglomerado Oriente de la ciudad (comunas 7, 13, 14, 15 y 21), el cual resguarda una gran población afrodescendiente, proveniente del afropacífico colombiano, producto de diferentes migraciones históricas y configuraciones espaciales en sus asentamientos de carácter popular (Urrea 2012), tal como se puede observar, señaladas en el círculo rojo, en la figura 1.

A nivel etnomusicológico, Luján (2015) propuso comprender procesos emergentes como las versiones locales del hip-hop bajo la noción de músicas turbias, debido a la precaución etnomusicológica que se debe tener con los orígenes étnicos, consumos culturales y demás esencialismos en el abordaje teórico de este tipo de músicas y fenómenos culturales. En la articulación conceptual planteada, una rama de la psicología musical se pregunta por la utilización de señales en la comunicación de emociones y estados anímicos en las prácticas musicales (Juslin 2000).

Estos estudios plantean la necesidad de com- prender los procesos de transmisión emotiva en grupos sociales a partir del descubrimiento de los efectos que producen en las personas, la escucha musical y la presencia de actos musicales en vivo. Algunos de estos mecanismos se establecen sobre la base del placer y el gusto en tanto dimensiones que influyen en la predicción de las emociones expresadas en la música (Ritossa y Rickard 2004).

En síntesis, podemos decir que muchas de las complejidades comunicacionales de los grupos sociales posibilitan apropiaciones culturales de carácter creativo, en algunos casos - tal como intentaremos demostrar - mediante la expresividad musical basada en sus posibilidades materiales y formas de comprender el mundo.

Estas apropiaciones se evidencian en el proceso de creación musical y cultural que permite la producción material de canciones, estilos de interpretación, performances y demás aspectos de expresividad identificados por el grupo como propios de una cultura 
específica por ejemplo, en el caso de los raperos de Cali dejar a un lado la apología a la violencia y el gansterismo usual en la estética hip-hop norteamericana (Vélez 2004, Luján 2016) discontinuidad que evidencia la necesidad de proponer un estilo propio de hacer y consumir música además de un fuerte carácter contestatario frente a la violencia sistemática ejercida hacia los jóvenes afro de sectores populares en la Cali de los noventa (Luján 2016).

Como complemento podemos decir que aspectos como el gusto, el placer, el origen etno-racial, el relacionamiento ideológico, la influencia, el habla, la emergencia de las escenas sociales o la percepción cognitiva vehiculan los comportamientos colectivos y establecen múltiples vínculos susceptibles de ser comprendidos a través de la noción de red social y las tecnologías investigadas alrededor de esta amalgama de técnicas (Reynoso 2011).

El esfuerzo que nos planteamos por comprender estas relaciones y sus dimensiones en la vida del colectivo de raperos de Cali motiva la realización de este estudio. La pregunta que guía esta investigación es la siguiente: ¿Cómo se puede establecer una red musical basada en los registros fonográficos de un grupo sociocultural determinado?

Como trabajo empírico se recopilaron y analizaron datos etnográficos y objetos materiales derivados de la escena rapera caleña y su historia a partir de diferentes perspectivas para la fecha indicada. En este espacio nos interesa visibilizar las relaciones de este conglomerado en términos de redes, como una base de trabajo objetiva y realizar el análisis correspondiente.

Como objetivo de análisis se eligieron las relaciones etno-raciales y de género como aspectos posibles de indagación con el fin de visibilizar su estructura sociocultural. Sabemos que otro tipo de relaciones también se pueden considerar como fundamentales en un grupo étnico determinado, pero dada la necesidad de establecer un conjunto de relaciones susceptibles de la comprobación científica a partir de los datos disponibles, se determinó emplear el análisis de las relaciones constitutivas del fenómeno presentado, mediante la indagación de las características distintivas - ya mencionadas - desde una perspectiva de red grupal o colectiva.

La relación que nos proponemos establecer a partir de los datos que disponemos, parte de la necesidad de visibilizar y analizar la red de raperos de la ciudad de Cali en la década del noventa, basándonos en la etnografía realizada sobre el acervo musical de este grupo sociocultural. En este espacio los criterios presentados para establecer la red propuesta se basan en las relaciones analizadas de los datos disponibles.

\section{METODOLOGÍA}

En la investigación, se realizó un abordaje etnográfico y etnomusicológico de la música rap de Cali en la década del noventa, con un enfoque reflexivo, desde una perspectiva investigativa de tipo experiencial. Este tipo de abordaje experiencial, el cual incluye la etnografía como técnica cualitativa de investigación, otorga las ventajas informativas de alguien que fue actor/agente de los fenómenos investigados alrededor del problema planteado. La reflexividad que propone el abordaje experiencial enfatiza el uso de la práctica y la rutina como marcador ontológico, enlazado con el conocimiento del lenguaje, de la cultura, del territorio y de las lógicas poblacionales evidenciadas en los aspectos considerados en el campo de problematización (Luján 2016).

Como resultado es escrutinio musical y experiencial estipulado estableció una base musical coherente de análisis con la producción sociocultural del grupo social abordado, sus consumos culturales, y sus producciones musicales. Este enfoque fue complementado por una indagación histórica, testimonial y bibliográfica del fenómeno considerado 
(Cuenca 2001, Luján 2016, Vélez 2004).

Por otra parte, el modelo investigativo empleado se basa en un enfoque mixto y experimental, en el sentido de la vinculación de aspectos músico-culturales juveniles con las métricas del análisis de redes sociales (Reynoso 2011). Esta apuesta innovadora reconoce la no existencia de un modelo previo similar, en la literatura disponible hasta la fecha, sobre una exploración de este tipo, lo cual reconoce las dificultades de establecer los aciertos y posibles equívocos esbozados en esta propuesta analítica.

Un segundo momento definió los dos tipos de redes presentadas a partir de los métodos que integran lo que Watts (2004) denomina "la nueva ciencia de las redes". El análisis de redes sociales o ARS posibilita establecer a partir del reconocimiento de nodos, vínculos y relaciones de diversos tipos y niveles, y una cadena de conexiones sociales que el fenómeno homínido del estar juntos posibilita.

De modo general podemos decir que el ARS permite identificar el tipo de arquitectura de una red social más allá de su estructura simple, ya que considera a partir de la teoría de grafos una serie de patrones, métricas, visibilizaciones y distribuciones en la indagación del tipo de complejidad que las redes presentan como características, la bibliografía al respecto es abundante (Barabási 2003; Carrington, Scott y Wasserman 2005; Carrington y Scott 2011; Fu et al. 2012; Kossinets y Watts 2009; Reynoso 2011; Taleb 2008; Watts 2004).

Tanto la etnomusicología como el ARS establecido en este espacio constituyen posibles ejercicios transdisciplinarios respecto al estudio de los grupos sociales. De manera muy reducida podemos decir que la primera estudia la música, sus relaciones sociales, productos culturales y sentidos en una comunidad determinada. Por su parte el ARS identifica y profundiza en las dinámicas del ensamblaje social y sus sistemas dinámicos $\mathrm{y}$ ofrece posibles explicaciones para conocer la realidad, ya que como podemos imaginarnos desafía cualquier tipo de sentido común.

En un nivel técnico, las redes establecidas fueron analizadas con la aplicación SocNetV v. 2.1. (Kalamaras 2015). Por último, el trabajo de campo en el cual se basa el modelo planteado se realizó durante los años 2014-2016 y se pudo validar su diseño en el terreno a través de retroalimentaciones presentadas por los artistas, además de los datos presentados en la investigación (Luján 2016) propuesta que conjuga una perspectiva tanto etic como emic, como se explicó anteriormente.

\section{Relacionamiento social a través de redes sociales}

En el proceso de visibilización de las redes sociales presentadas en este trabajo es pertinente tener en cuenta que la figura 2 presenta el tipo de relacionamiento entre los nodos (grupos de rap, ver tabla 2) "Código" y "Los Generales", la conexión étnica y musical (dirigida) en tanto escena cultural se establece a partir de un relacionamiento de tipo "afro-mestizo", lo cual fue evidenciado a partir del trabajo de campo y los datos musicológicos (Luján 2016). Para esta labor se almacenaron los datos entre nodos (grupos de rap) y arcos (vínculos dirigidos) de la siguiente manera:

$$
\begin{aligned}
& \text { *Nodos (Vértices) } 2 \\
& 1 \text { "Código Rap" } \\
& 2 \text { "LosGenerales" } \\
& \text { *Arcos (Arcs): 1 "etnicidad" } \\
& 12 \text { c red 1 "afro-mestizo" }
\end{aligned}
$$

Debido a que el problema presentado contiene un carácter histórico-cultural, fue necesario el análisis de una extensa documentación, incluido un conjunto de álbumes musicales que pudieron ser recuperados de los archivos de coleccionistas de música hip-hop de la ciudad de Cali y de los propios artistas con los cuales se tuvo contacto, lo cual permitió establecer un base sociocultural (material) definida y demarca de manera clara los inicios organizativos y de movilización de esta 
escena (Luján 2016).

En la investigación efectuada, se profundizó en el tipo de complejidad de la red estudiada, a partir de identificar sus características y relacionar la idea de complejidad correspondiente a un grupo sociocultural basado en un eje nodal-musical específico; la música rap y la cultura hip-hop.

Para lograr este cometido se establecieron dos tipos de redes, una red étnica, denominada red 1, que se puede apreciar en la figura 2 y una red de género (red 2), a partir de dos tipos de categorías básicas: afro-mestizo y masculino-femenino. Los pesos de los nodos fueron creados conforme el número de integrantes de cada agrupación, y el orden jerárquico de aparición de cada nodo fue situado de modo aleatorio.

Consideramos que este ejercicio de investigación transdisciplinaria es una apuesta a la consolidación de unas ciencias sociales complejas en las cuales se presenten posibilidades de configuración investigativa acentuadas en las nuevas tecnologías disponibles y algunos abordajes exploratorios recientes.

En la figura 2, los colores introducidos refirman el potencial de identificación de los tipos de relaciones étnicas de los jóvenes afro raperos de la ciudad de Cali en el periodo de los años noventa y de manera incidental contribuyen a la distinción de diferentes actores en este caso nodos los cuales se relacionan de manera directa representado a través de arcos (con flechas) en los diferentes escena- rios del universo de la cultura hip-hop local; los espacios de creación cultural, difusión musical, círculos de producción musical y demás alternativas de cohesión social y relacionamiento.

El tipo de representación empleada es de tipo colectivo y no obedece a redes personales o grupales (individuales), ya que la naturaleza del caso estudiado se concibió en dos fases. La primera introdujo los tipos de relaciones étnicas a partir de las identificaciones etnoraciales en las cuales la identificación del hip-hop juega un papel importante debido al reconocimiento de la cultura hip-hop como un fenómeno de filiación étnica afroamericano, sin embargo el carácter etnoracial de los miembros de los grupos establecidos posibilitó una presunta identificación sociocultural objetiva, lo cual introduce una perspectiva de apropiación etno-simbólica (Gans 1979) que orienta el análisis.

Sobre los mismos presupuestos la segunda fase identificó la visualización descriptiva de las relaciones de género a través del establecimiento de una red de este tipo. Como es usual en este tipo de análisis, las métricas presentadas intentan describir el nivel de relacionamiento - o nivel de complejidad - de la escena musical considerada estableciendo un tiempo y espacio específico.

Figura 2: Red 1 visualizada como centralidad de poder circular

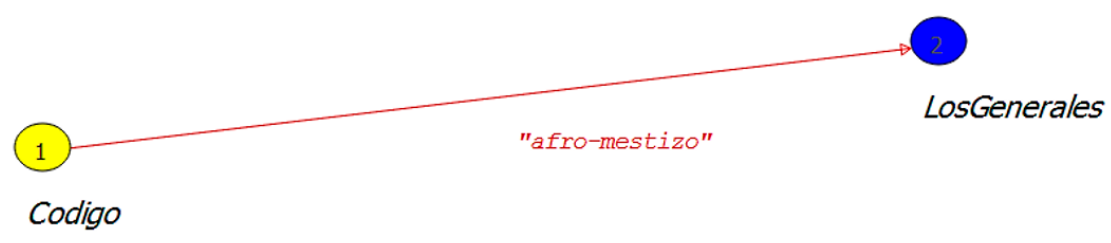

Fuente: elaboración propia. 


\section{RESULTADOS Y DISCUSIÓN}

\section{Etnicidad y raperos en la ciudad de Cali}

Producto de la investigación, se determinó el surgimiento de la escena rapera en la ciudad de Cali, a partir de un proceso de comunicación interna y transversal, en el cual los miembros de este conglomerado estuvieron conectados a partir de diferentes tipos de relaciones; amistad, filiaciones étnicas, influencia del mercado musical internacional, auto-reconocimiento, procesos identitarios y sentido de pertenencia territorial e identificaciones, como algunas de las escasas investigaciones sobre este fenómeno así lo plantean desde diferentes perspectivas (Cuenca 2001, Luján 2016, Vélez 2004).

En tal sentido, la comunicación entre diferentes tipos de redes humanas implica el reconocimiento de roles y diferentes aspectos derivados de reconocimiento mutuo y la tolerancia. En un nivel micro a propósito de una comunidad determinada, los raperos de Cali emplearon la música como un eje nodal juvenil para la creación de su discursividad y planteamientos liricales.

Una perspectiva meso o de ciudad, indica las poblaciones afro o negra e indígena son las más desventajadas en la sociedad caleña si se comparan la población mayoritaria sin auto-reconocimiento étnico, debido a una mejor situación económica, educativa, de inserción en el mercado laboral, de salud y de movilidad intracitadina (Urrea 2012).

Una tercera escala macro introducida sobre la región latinoamericana establece sobre las sociedades latinoamericanas una perspectiva similar denominada "pigmentocracia social" (Telles 2014), lo cual indica una suerte de sociedad rankeada a partir del color de la piel y establecida sobre grupos sociales - y su componente étnico-racial - en diferentes situaciones diferenciadas de poder asimétrico.

Esta serie de hallazgos sobre la comunidad afro de la ciudad de Cali y las comunidades afrolatinoamericanas indican la necesidad de focalizar sus estrategias de organización social al lado de las ideas clásicas de las ciencias sociales en las cuales lo económico, lo político y lo cultural en muchas ocasiones se presenta de manera reducida al análisis basado en indicadores de la organización social y las formaciones históricas de los grupos humanos y su conformación del territorio geográfico.

Por esta razón, el foco del análisis presentado se establece sobre las dinámicas de la red de un grupo juvenil (para la época de la década del noventa) y su producción sociocultural. Así, la comunicación músico-juvenil posibilita la ampliación de las estrategias de consolidación de una escena emergente en circunstancias de desigualdad económica y desventajas acumuladas históricamente (Urrea 2012).

\section{La red caleña de raperos al finalizar el mi- lenio}

Como ya se explicó las redes propuestas para visualizar este estudio, se establecen a partir de la comunidad caleña de raperos al finalizar el milenio. La primera red presentada, que se puede apreciar en la figura 3, se basa en las relaciones de etnicidad evidenciadas en los raperos de Cali, aunque es pequeña ya que presenta solo 15 nodos, los vínculos y relaciones se expanden mucho más, figurando en total 105, con una densidad de 1 . La segunda red, que se aprecia en la figura 4), es igualmente pequeña con un total de 15 nodos, pero a diferencia de la primera red presenta 207 vínculos con una densidad más baja de 0.98571 .

En el análisis de ambas figuras, se precisa que están fuertemente conectados, ya que su característica de alta densidad ratifica este rasgo distintivo, para su elaboración se utilizó como fuente primaria la indagación y escucha de los trabajos musicales recolectados sobre la población estudiada y sus tipos colaboraciones, y se propuso establecer las vinculaciones que las conforman. 
Tabla 1: Algunas medidas consideradas en la comparación entre ambas redes

\begin{tabular}{ll}
\hline \multicolumn{1}{c}{ Red 1 } & \multicolumn{1}{c}{ Red 2 } \\
\hline Tipo de grafo: no dirigido está conectado. & Tipo de grafo: dirigido fuertemente conectado. \\
Diámetro: 0. & Diámetro: 2. \\
Excentricidad $(e)$. Max e $=0.5$ (nodo 4) & Excentricidad (e). Max e =2 (nodo 14) \\
Min e $=0.0909091$ (nodo 3) & Min e $=1$ (nodo 1) \\
Clases de $e=7$. & Clases de $e=2$. \\
Coeficiente de clustering local promedio $(G C L C)=0.6$. & Coeficiente de clustering local promedio $(G C L C)=0.98681$. \\
El nodo 4 tiene máximo coeficiente de clustering: 1. & El nodo 13 tiene máximo coeficiente de clustering: 1. \\
El nodo 1 tiene el minino coeficiente de clustering: 0. & El nodo 1 tiene el minino coeficiente de clustering: 0.98352. \\
Rango: $0<G C L C<1$. & Rango: $0<G C L C<1$. \\
Max grado de centralidad $(D C)=0.18033$ (nodo 3). & Max grado de centralidad $(D C)=1$ (nodo 1).. \\
Min DC' $=0.032787$ (nodo 4). & Min $D C=0.85714$ (nodo 15). \\
Clases de DC $=15$. & Clases de $D C=15$. \\
DC sum $=854$. & DC sum $=207$. \\
DC sum $=1$. & DC sum $=14.786$. \\
Este grafo esta ponderado y no presenta valor de Grados de & Grados de centralización grupal (GDC) $=0.0010933$. \\
centralización grupal (DGC). & \\
\hline
\end{tabular}

Fuente: elaboración propia.

La tabla 1 presenta la comparación de algunas mediciones basadas en los atributos de las dos redes presentadas; tipos de grafos, diámetros de las redes, excentricidad (lo cual es una medida de integración de la red), coeficiente de clustering, máximo grado de centralidad y grados de centralización grupal.

El índice de coeficiente de clustering local promedio (GCLC) - o coeficiente de agrupamiento - precisa que su valor es 0 cuando la estructura de la red no presenta cliques (el mayor conjunto de entidades conectadas - vértices - a otros elementos de la red, es decir un subgrafo.

Por ejemplo, un árbol acíclico, en cambio su valor es 1 (como la red 2 y seguido por la red 1), cuando cada nodo y su vecino son clicas completas. La fórmula matemática del GCLC utilizada en el algoritmo de SocNetV se expresa como sigue para una grafo dirigido $\mathrm{Ci}=\mid\{$ ejk : vj,vk $\square \mathrm{Ni}$, ejk $\square \mathrm{E}\} \mid$ $\mathrm{ki}(\mathrm{ki}-1)$, y para un grado no dirigido $\mathrm{Ci}=2$ |\{ejk : vj,vk $\square \mathrm{Ni}$, ejk $\square \mathrm{E}\} \mid \mathrm{ki}(\mathrm{ki}-1)$, donde $\mathrm{Ci}$ es el coeficiente de clustering, ki el nú- mero de vértices, $\mid \mathrm{Ni}$, en la vecindad $\mathrm{Ni}$ de un nodo i.

Se hace necesario realizar la siguiente consideración. En los últimos tiempos algunos investigadores consideran diferentes tipos de distribuciones en las redes complejas, tanto en las distribuciones de la familia Gaussiana (Taleb 2008) como en los diferentes tipos de distribuciones de las ciencias de la complejidad. Estas consideraciones conllevan a profundas implicaciones de tipo epistemológico, dado que el asunto es bastante denso para tratarlo en este espacio el lector puede dirigirse al trabajo de Taleb (2008) y Reynoso (2011) sobre este asunto en particular.

Para continuar el análisis, la idea de clustering o GCLC hace alusión al conglomerado que indica la probabilidad de vinculación entre dos vecinos de un nodo, por lo tanto a mayor clustering mayor cercanía, ya que aparecen menores conglomerados en la red. Sobre los grados de centralidad grupal GDC se dice que es la medida que cuantifica cuántos lazos con vínculos tiene un nodo con otros nodos en la red. 
El formalismo se presenta a partir de la fórmula de Freeman la cual se presenta de la siguiente manera: $\mathrm{GDC}=\sum\left(\operatorname{maxDC} \mathrm{C}^{\prime}-\mathrm{DC}^{\prime}\right)$ / (N-1) $\square(\mathrm{N}-2) /(2 \square \mathrm{N}-1)$, donde el máximo grado de centralización grupal de cada nodo o (vértice) u es la cantidad de arcos o aristas que se le atribuyen. Esta medida en el análisis que nos compete indica el número de enlaces de un grupo de rap con los demás que conforman la red.

La comparación entre ambas redes indica que las dinámicas de género y su relación con lo femenino tuvieron un mayor impacto que las perspectivas de diferenciación racial, ya que el diámetro de la red 1 es 0 y el de la red 2 es 2, también las clases de excentricidad e revelan en el primer caso 7, y para el segundo sólo 2, es decir una menor distancia. Se debe reconocer que esta descripción se manifiesta sobe la base de los álbumes creados, pero no recoge la totalidad de las canciones producidas por los raperos caleños hasta la década del noventa.

La excentricidad e o el número de asociación de cada nodo u, el cual es la mayor distancia geodésica entre ese nodo y cada otro nodo de la red es decir, esta medida estipula la lejanía de cada nodo con otro nodo, en nuestro caso que tan lejos estaba cada grupo de rap con su símil.

El formalismo propuesto es $\epsilon \mathrm{U}=\max \{\mathrm{d}(\mathrm{u}, \mathrm{v})$, $\mathrm{Vv} \in \mathrm{V}\}$, donde la e o el número de asociación de cada nodo u es la mayor distancia (d) geodésica entre ese nodo y cada otro nodo $(\mathrm{u}, \mathrm{v})$ de la red. Esta distancia de tipo geodésico o topológico indica entre ambas redes estudiadas un minino de 0 pasos entre un grupo y otro, y un máximo de 2 pasos para establecer cualquier tipo de relación. Lo que significa en la red 1 un vínculo de índole étnica, pero en la red 2 una posible relación de género. En términos de la fluidez de la comunicación como reconoce Cárdenas:
A mayor distancia, más extensa es la red y más costosa es la comu- nicación y la posibilidad de coor- dinación. A menor distancia, más compacta es la red y más fácil es la comunicación y coordinación ya que se debe recurrir a menos conectores intermediarios. (Cárdenas 2017:79)

Figura 3: Red 1 visualizada como centralidad de poder circular

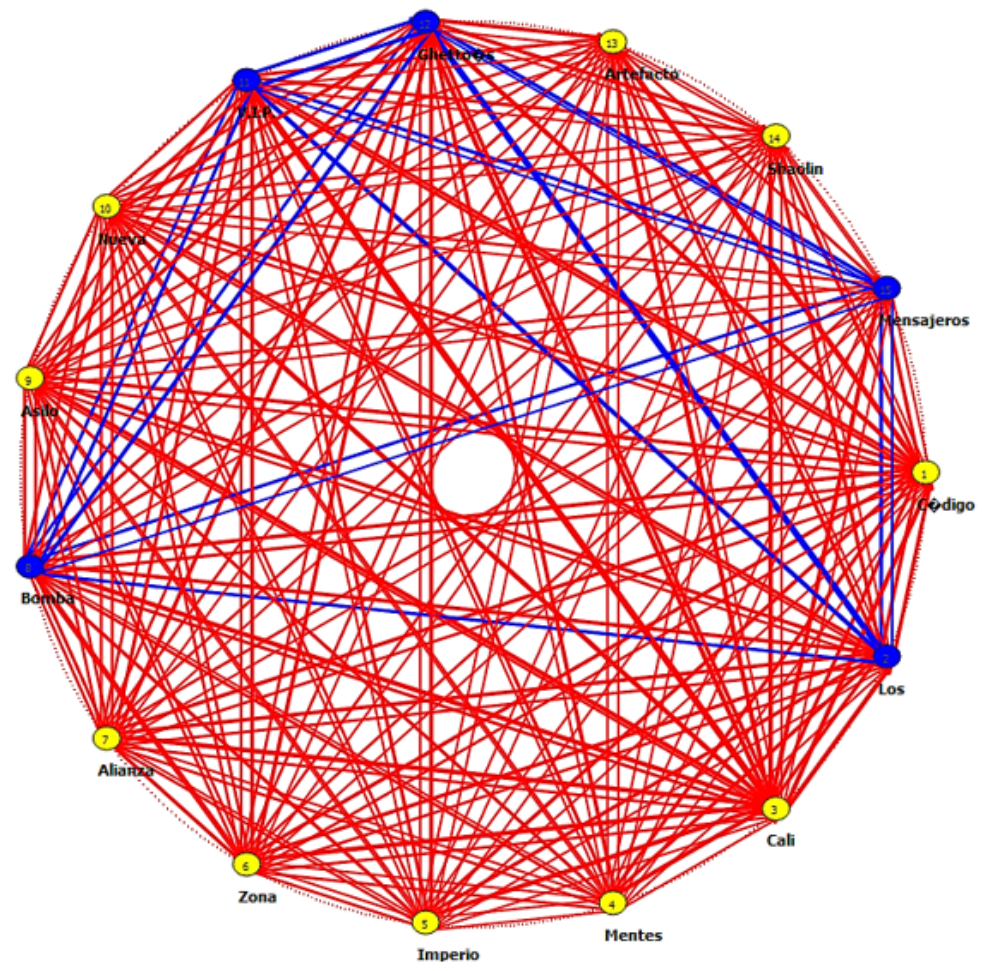

Fuente: elaboración propia.

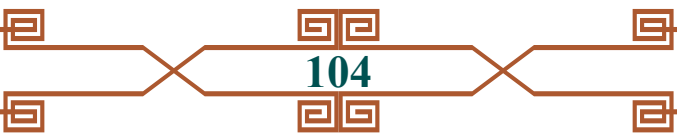


Los números de identificación de la red se establecieron a partir del año de lanzamiento de los discos.

- Relación "afro-afro"

- Relación "afro-mestizo" (las descripciones grupales se encuentran en la tabla 2)

Los números de identificación de la red se establecieron a partir del año de lanzamiento de los discos.

- Relación "masculino-masculino" (ver el nombre de los nodos o grupos en letra verde) - Relación "masculino-femenino" (las descripciones grupales se encuentran en la tabla 2)

En ambos casos, la complejidad de las redes abordadas es baja, ya que la cantidad de sus nodos no es extrema y tampoco sus vínculos. En la red 1 (ver figura 3), los actores establecen relaciones étnicas entre el carácter "afroafro" (líneas de color azul) y "afro-mestizo" (líneas de color rojo), esta última relación es preponderante en la red.

La red 2 (ver figura 4), presenta la relación "masculino-masculino" y "masculino-femenino" en la cual como se puede comprobar predomina la relación "masculina", los nodos de color azul incluyen mujeres en su conformación grupal (María Morocha de "Bomba de Tiempo" y Natrix de "Alianza y Colombia Rap Cartel").

Es importante decir que al finalizar la década de los noventa fueron muchas las raperas que se mantuvieron activas en la ciudad de Cali, por ejemplo, el grupo la Colonia o el grupo Esfinges, pero lastimosamente no produjeron álbumes o lanzaron discos formales en formato CD, Lp, o cassette, sin duda ambos grupos fueron protagonista en esta escena.

A partir de lo anterior no podemos concluir que la cultura hip-hop en la Cali de los noventa fuera machista o segregacionista desde una perspectiva de género, pero se evidencia una inequidad sobre este aspecto puntual, esto quizá debido a factores culturales de la época, la imagen de la cultura hip-hop norteamericana y sus imaginarios de misoginia, o quizá debido a la rudeza de la estética hiphop.

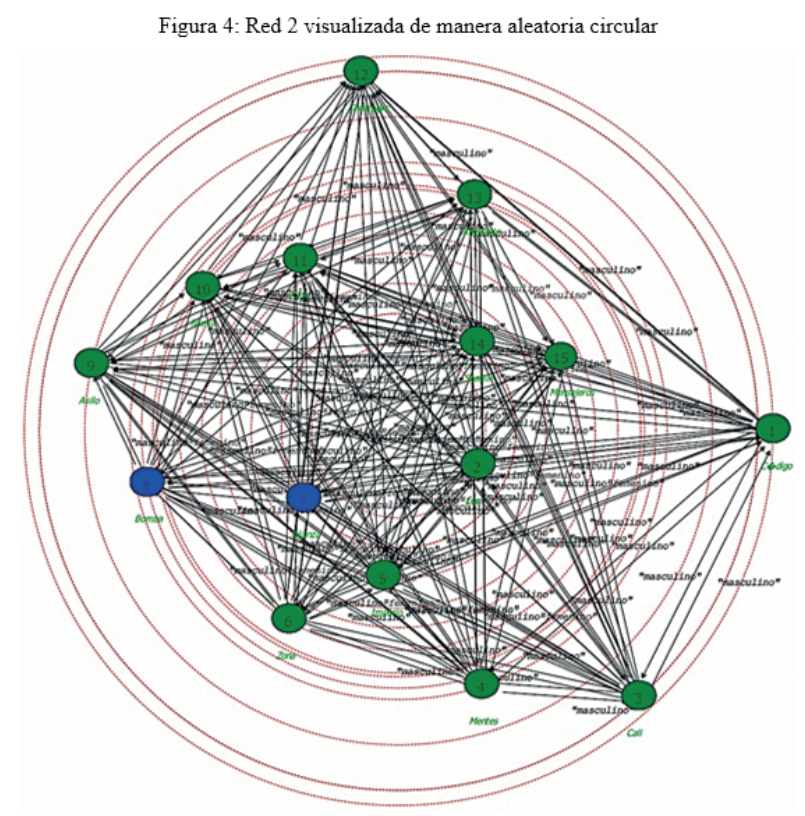

Fuente: elaboración propia 
Tabla 2: Producciones de rap en Cali en los años 90 hasta el año 2003

\begin{tabular}{|c|c|c|c|c|}
\hline Grupo e Integrantes & $\begin{array}{l}\text { Álbum y } \\
\text { formato }\end{array}$ & Sello & $\begin{array}{c}\text { Ubicación Espacial } \\
\text { (Barrios) }\end{array}$ & Año \\
\hline $\begin{array}{l}\text { Código Rap } \\
\text { (James, Juan José, Carlos Catacolí, Jhonny, Pablo, } \\
\text { Diego D.J.) }\end{array}$ & $\begin{array}{l}\text { Fuera de la Fila } \\
\text { (Sencillo) }\end{array}$ & $\begin{array}{l}\text { Codiscos } \\
39805232\end{array}$ & Sin datos & 1991 \\
\hline $\begin{array}{l}\text { Los Generales R\&R } \\
\text { (Jhonky Barry (q.e.p.d.), Jhonny Jein, Propio Way, } \\
\text { Rauliman, Chiqui Bryan, Freddy Willian) }\end{array}$ & Sin datos & Sin datos & Buenaventura/Cali & $\begin{array}{l}\operatorname{Sin} \mid \\
\text { datos }\end{array}$ \\
\hline $\begin{array}{l}\text { Cali Rap Cartel } \\
\text { (Clave Latina, New Power, Primera Fase, Nueva } \\
\text { Sentencia, Farsantes del Rap, M.C. Brayan \& Dilurri } \\
\text { M.C. y Doble Identidad. }\end{array}$ & Sin datos & UNIVALLE & Sin datos & 1996 \\
\hline $\begin{array}{l}\text { Los Generales } \\
\text { (Propio Way, Bongo Man, O.P.P., Candy Man, } \\
\text { Freddy Willian, Harry Boom, Antonio) }\end{array}$ & $\begin{array}{l}\text { Coge a tu negro } \\
\text { (C.D.) }\end{array}$ & PTD 1433-96 & Buenaventura/Cali & 1996 \\
\hline $\begin{array}{l}\text { Mentes Oscuras } \\
\text { (Fénix Waira, Tanatos) }\end{array}$ & $\begin{array}{l}\text { La Divina } \\
\text { Comedia } \\
\text { (casete) }\end{array}$ & Mentes Oscuras & El Poblado/ & 1997 \\
\hline $\begin{array}{l}\text { Imperio } \\
\text { (Mae, Puto, Kruell) }\end{array}$ & $\begin{array}{l}\text { Septiembre } 8 \\
\text { (casete) }\end{array}$ & $\begin{array}{l}\text { Suicidio } \\
\text { Producciones }\end{array}$ & Paraíso/Salomia & 1998 \\
\hline $\begin{array}{l}\text { Zona Marginal } \\
\text { (Blade-Fer Shaolin, Rico, Jhon J) }\end{array}$ & $\begin{array}{l}\text { La Expresión de } \\
\text { Un Pueblo } \\
\text { (C.D.) }\end{array}$ & $\begin{array}{l}\text { Zona } \\
\text { Producciones }\end{array}$ & $\begin{array}{l}\text { La Unión de Viviê๋nda } \\
\text { Popular/ Alfonso Bonilla } \\
\text { Aragón }\end{array}$ & 1999 \\
\hline $\begin{array}{l}\text { Alianza NRP } \\
\text { (Natrix, Rocky, El Paisa) }\end{array}$ & $\begin{array}{l}\text { Colombianos de } \\
\text { Barrio (D.C.) }\end{array}$ & Sin datos & (Medellín/Cali) & 1999 \\
\hline $\begin{array}{l}\text { Bomba de Tiempo } \\
\text { (Gallito, Julio, Morocha, Gerardo) }\end{array}$ & (casete) & Sin datos & El Retiro & 1999 \\
\hline $\begin{array}{l}\text { Imperio } \\
\text { (Mae, Puto, Kruell) }\end{array}$ & $\begin{array}{l}1460 \quad \text { Días } \\
\text { Infernales } \\
\text { (C.D.) }\end{array}$ & $\begin{array}{l}\text { Suicidio } \\
\text { Producciones }\end{array}$ & Paraíso/Salomia & 1999 \\
\hline $\begin{array}{l}\text { Asilo } 38 \\
\text { (Al Roc, Rocky, Cap, D.R. Ganja, Smoka) }\end{array}$ & $\begin{array}{l}\text { La Hoguera } \\
\text { (C.D.) }\end{array}$ & $\begin{array}{l}\text { Cap } \\
\text { Producciones }\end{array}$ & $\begin{array}{l}\text { San Pedro/El } \\
\text { Vergel/Conquistadores } \\
\text { (Cali/Bogotá) }\end{array}$ & 2000 \\
\hline $\begin{array}{l}\text { Cali Rap Cartel } \\
\text { (Jhon J, Menester, Judio, Orador M.D.C. Detective, } \\
\text { Enigma, Pife (q.e.p.d.), Jhaga, Shedda, Rico, Blade- } \\
\text { FerShaolin) }\end{array}$ & $\begin{array}{l}\text { Armada } \\
\text { Zomacimen } \\
\text { (cassette) }\end{array}$ & $\begin{array}{l}\text { CRC } \\
\text { Productores }\end{array}$ & $\begin{array}{l}\text { El Poblado, El Retiro, La } \\
\text { Unión de Vivienda Popular, } \\
\text { Alfonso Bonilla Aragón, } \\
\text { Antonio Nariño, El Vergel, } \\
\text { República de Israel, } \\
\text { Marroquín }\end{array}$ & 2000 \\
\hline $\begin{array}{l}\text { Cali Rap Cartel } \\
\text { (Jhon J, Menester, Judío, Orador M.D.C. Detective, } \\
\text { Enigma, Pife (q.e.p.d.), Jhaga, Shedda, Rico, Blade- } \\
\text { Fer Shaolin) }\end{array}$ & $\begin{array}{l}\text { Cali Rap Cartel } \\
\text { Parte I } \\
\text { (C.D.) }\end{array}$ & $\begin{array}{l}\text { CRC } \\
\text { Productores }\end{array}$ & $\begin{array}{l}\text { El Poblado, El Retiro, La } \\
\text { Unión de Vivienda Popular, } \\
\text { Alfonso Bonilla Aragón, } \\
\text { Antonio Nariño, El Vergel, }\end{array}$ & 2000 \\
\hline
\end{tabular}




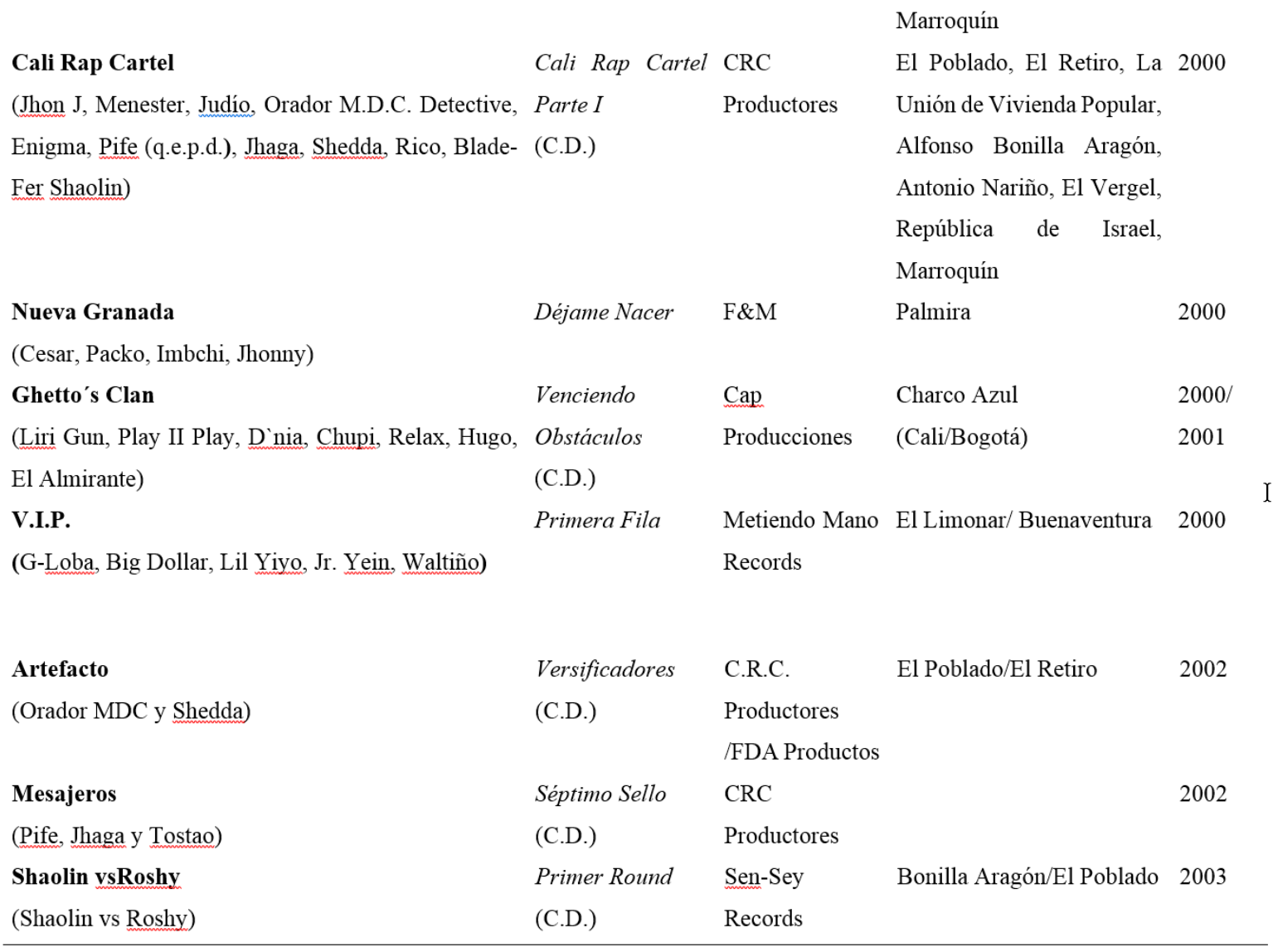

Fuente: elaboración propia.

Sí consideramos los insumos musicales de los cuales disponemos, los álbumes recopilados en esta informe - tal como figura en la tabla 2 - constituyen la mayor parte del rap producido en Cali para la fecha, ya que existe una gran cantidad de "demos" o canciones que fueron producidas pero no formalizadas en un disco, álbum o cinta-cassette, la muestra recogida es significativa, ya que abarca los discos que fueron elaborados en su totalidad lo cual fue corroborado en el trabajo de campo, en una fase posterior en la cual se presentó el informe final de investigación ante algunos de los protagonistas de este escena musical en la ciudad de Cali.

A nivel musical, la mayoría de las canciones son rap clásico, escrito en compases de 4/4, con downtempos de 90 a 120 bpm, donde se utilizaron samples de otras músicas. Tan solo el grupo Los Generales incursionó en el merengue y el reggae. El grupo Alianza que trabajo el disco "Colombianos de barrio" utilizó musicalización en vivo, y se incluye en este conglomerado por la participación del rapero caleño afro Rocky fundador del colectivo Cali Rap Cartel y Asilo 38 quien también colaboró con el grupo Poetas de la Oscuridad. El abordaje experiencial al cual se hizo referencia arriba hizo posible la comprobación de estos datos y su refrendación se realizó durante una visita a terreno realizada en diciembre de 2015 (Luján 2016).

En nuestro caso específico, la interacción entre todos los componentes de las redes configuradas en los grupos de rap y sus relaciones, demuestra cómo los lazos de etnicidad comprendidos como el uso de la simbología propia de la cultura hip-hop, la auto-identificación como raperos y afros, la identificación con las problemáticas derivadas de la violencia sistemática hacia jóvenes de sectores populares caleños (Luján 2016) y las relaciones de género a partir de los musical, antes de ser esencializados problematiza las 
vínculos simbólicos y materiales del trabajo cultural.

Es sabido que la música rap y hip-hop descienden de sectores populares marginalizados y se configuran desde una fuerte base genealógica y étnica afro, pero esto no excluye que los contextos mediante los cuales se desenvuelve la cultura hip-hop presente apropiaciones globales y locales en los nichos donde se configuran a nivel planetario (Mitchell 2001) y en continentes como África (Charry 2012) y Sudamérica y el Caribe (Pardue 2014).

El caso de del rap de Cali a finales del siglo pasado orquesta un proceso comunicativo de apropiación local del hip-hop no en términos musicales, mejor como proceso de "etnicidad simbólica". A propósito de la internacionalización del hip-hop (Mitchel 2001), desde los procesos locales y emergentes se evidencia una clara homofilia en el sentido del establecimiento de códigos compartidos, la incorporación de elementos comunes como los performances, vocablos, estéticas particulares y comunicaciones corpóreas que pueden operar de manera interna o intragrupal y transversal o globalizada.

Experiencias compartidas como la exclusión social, la pobreza material extrema, la discriminación sociorracial y étnica entre otros factores que aquejan las juventudes y poblaciones latinoamericanas especialmente afro e indígenas contemporáneas (Telles 2014) también generan movilizaciones en las cuales las expresividades actuales y ancestrales juegan un rol determinante.

Gans (1979), propuso la noción de etnicidad simbólica para explicar las formas en las cuales existe una fuerte identificación en los ascendientes de inmigrantes - basándose en el caso de los norteamericanos (judíos o italianos)-, con nuevos modos de etnicidad, quienes necesitarían de organizaciones y culturas, y demás resortes étnicos simbólicos para solidificarse.
La identidad para Gans (1979) no puede existir por fuera de un grupo. Más allá, de una "línea directa" que configura la etnicidad, el sociólogo se preguntaba también por los descendientes de tercera y cuarta generación que reclamaban como propio - a propósito del caso judío-, los dos tipos de culturas propias, o mejor, las formas tanto "seculares como sagradas" de su cultura (Gans 1979).

Para el caso de los raperos a nivel planetario las experiencias de segregación social y especial motivaron escenarios de movilización en los cuales la música y lo estético revindicaron luchas sociales en tanto alternativas culturales como reconoce Mitchell (2001) para el caso colombiano.

Es importante recordar que los resultados presentados en este estudio se basan en su totalidad en datos históricos (Luján 2016). Así, las redes establecidas a partir de aspectos como el grupo étnico, canciones, álbumes, estilos o número de miembros, evidencian la configuración de un acervo musical del rap en la Cali de los años noventa, legado que se puede observar hoy en día en la ciudad.

Por otro lado, la interpretación de los datos empíricos nos dice que ambas redes representan parte de las dinámicas colectivas del fenómeno rapero caleño y una posible comunicación interétnica al servicio de la cultura hip-hop, ésta última conformada por el breakdance, la música rap, la práctica $\mathrm{DJ}$, el graffiti y su estética visual urbana y la producción musical.

El fenómeno también se comprende como un espacio fuertemente masculino, pero abierto a diferentes perspectivas dialógicas. Es preciso decir que no se consideraron para la creación de las redes visibilizadas los breakdancers (bailarines de break dance), DJ's, grafiteros o productores de rap en Cali para la época, ya que el foco se centró en la música grabada por la escena emergente de raperos en su mayoría afrodescendiente como se pudo comprobar. 


\section{CONCLUSIONES}

Hemos presentado la visualización y análisis de las relaciones étnicas y de género de los jóvenes afro raperos de la ciudad de Cali en la década de los años noventa, a partir de un ejercicio de aplicación de ARS. Las dinámicas de ambas redes y su estructura social fueron estudiadas como redes grupales centrales no egocéntricas, bajo la perspectiva de explorar los constructos comunicativos posibles de sus vínculos.

El caso estudiado abre la posibilidad a mayores exploraciones en este sentido, pero la dificultad de la adquisición de los datos recaudados, ya que nos situamos en un caso de hace tres décadas aproximadamente, permite la postulación concreta de los resultados presentados en este espacio.

El estudio efectuado integró las perspectivas de transdisciplinariedad desde el aparato analítico de las redes sociales, las perspectivas de la complejidad y la etnomusicología en el abordaje de los datos presentados, con lo que se estableció la relevancia del fenómeno, considerado en un mundo cada vez más conectado y por lo tanto con nuevas y diferentes modalidades comunicativas en el sentido de la socialización cultural - en nuestro caso - de la música rap y la cultura hip-hop.

El análisis de las relaciones raciales y de género y la indagación de sus características distintivas desde una perspectiva de red grupal o colectiva, permitió identificar las posibles formas de relación existentes para esta comunidad agobiada por la violencia estructural, la falta de oportunidades laborales, la segregación socioespacial y la situación de marginalización histórica en la ciudad de Cali, una ciudad "con el mayor número de habitantes (en el país) que se autorreconocieron en el censo en alguna de las categorías como gente negra" (Urrea, 2012:27) según el censo nacional del año 2005.
Consideramos que una posible etnomusicología de la complejidad exige establecer los mecanismos comunicacionales de la cultura en apariencia inextricables en cada grupo social, esta premisa la habían considerado desde hace más de 60 años atrás Ruesch y Bateson cuando discurrían la noción de red cultural y el problema de la comunicación desde una perspectiva compleja:

La red de comunicación más compleja la hallamos cuando consideramos una red cultural en la que muchas personas se comunican con muchas otras. En ella tanto el mensaje como el destino del mensaje permanecen anónimos: esto hace imposible su corrección. (...) El conocimiento de estos puntos fijos dentro de la red de comunicación, permitiría a los participantes modificar, por medio de la realimentación, sus propios mensajes e interpretar correctamente los de los otros. (...) La mayor parte de estos enunciados abarca lo que la gente hace y dice, ha hecho y ha dicho. Es imposible observar directamente una "cultura" de este tipo: existe solamente una forma de enunciados generales que los estudiosos formulan acerca de la gente. Dichos enunciados incluyen no solo las normas de organización específicas de los grupos de personas sino también sus problemas jurídicos y económicos, su lenguaje y sistemas de simbolización, sus convenciones y tradiciones y todos aquellos objetos, construcciones y monumentos que transmitan mensajes del pasado. (Ruesch y Bateson 1965:38-39)

La agudeza de estas observaciones pone de relieve la red de significados compartidos a la cual podemos llamar cultura - en el caso que estudiamos - manifestada como una posible etnicidad simbólica de apropiación musicocultural, que penetrada en las paradojas de la comunicación cultural, posibilita lo que consideramos una relación de homofilia dibujada a partir de lo musical juvenil, y dinamizada sobre la base de una cultura mundial para la época en expansión, lo cual 
delinea el marco o framework de una posible comprensión de la complejidad cultural. La salvedad epistemológica propuesta por Ruesch y Bateson delimita el problema investigativo como una posibilidad más, entre $\mathrm{n}$ problemas a investigar respecto a lo cultural y la comunicación tejida en torno a ello. Quizá esta imposibilidad de corrección en los procesos sociocomunicativos sea la principal paradoja de un mundo cada vez más conectado a unos pocos nodos y vínculos de distancia.

El caso de los jóvenes afro de la ciudad de Cali al finalizar el milenio pasado, evidencia las maneras diversas de cómo las personas establecen vínculos de identificación más allá del contacto personal, ya que desde una perspectiva general, el carácter de las apuestas sonoro-estéticas y por supuesto musicales contemporáneas se puede concebir como transacciones sonoras (Luján 2015), es decir, re-apropiaciones, elaboraciones socioculturales experimentales, emplazamientos y demás resignificaciones trasladadas a y desde diferentes latitudes.

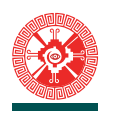

\section{REFERENCIAS BIBLIOGRÁFICAS}

Axelrod, R. (1997). The dissemination of culture: A model with local convergence and global polarization. Journal of Conflict Resolution, 41(2), 203-226.

Barabási, A. (2003). Linked: How everything is connected to everything else and what it means. Nueva York, Estados Unidos: Plume Books.

Cano, P. \& Markus, K. (2004). The emergence of complex networks patterns in music artist networks. En X. Serra (ed.). Proceedings of the 5th International Symposium on Music Information Retrieval (ISMIR 2004), (pp. 466-469). Barcelona, España: Universitat Pompeu Fabra.
Cano, P., Celma, O. \& Koppenberger, M. (2006). Topology of music recommendation networks. Chaos: An interdisciplinary journal of nonlinear science, 16 (1), s.p.

Cárdenas, J. (2017). Las redes de la elite académica de la Sociología. Revista Española de Sociología, 26 (1), 69-84.

Carrington, P., Scott, J. \& Wasserman, S. (2005). Models and Methods in Social Network Analysis. Cambridge, Reino Unido: Cambridge University Press.

Carrington, P. \& Scott, J. (2011). Handbook of Social Network Analysis. Londres, Inglaterra: Sage.

Charry, E. (2012). Hip Hop Africa: New African Music in a Globalizing World. Bloomington, Estados Unidos: Indiana University Press.

Chemillier, M. (2002). Ethnomusicology, ethnomathematics. The logic underlying orally transmitted artistic practices. En G. Assayag, H. Feichtinger \& J. Rodrigues (comps.). Mathematics and Music, (pp. 161-183). Nueva York, Estados Unidos: Springer.

Cuenca, J. (2001). La construcción de identidades sociales en un grupo de raperos (Tesis de maestría inédita). Universidad del Valle, Cali, Colombia.

Finnegan, R. (1992). Oral traditions and the verbal arts. A guide to research practices. New York, Estados Unidos: Routledge.

Fu, F., Nowak, M., Christakis, N. \& Fowler, J. (2012). The Evolution of Homophily. Scientific Reports, 2, 1-6.

Gans, H. (1979). Symbolic Ethnicity: The Future of Ethnic Groups and Cultures in America. Ethnic and Racial Studies, 2 (1), 1-20.

Headland, T., Pike, K. \& Marvin, H. (1990). Emics and Etics: The Insider/Outsider Debate. Newbury Park, Estados Unidos: Sage Publications.

IDESC. (2017). Infraestructura de datos espaciales, Santiago de Cali. Recuperado de http://idesc.cali.gov.co/ geovisor.php?id_mapa_fijar $=36$

Juslin, P. (2000). Cue utilization in communication of emotion in music performance: Relating performance to perception. Journal of Experimental Psychology: Human Perception and Performance, 26 (6), 1797-1813.

Kalamaras, D. (2015). Social Network Visualizer (SocNetV) - Social Network Analysis and Visualization Software. Recuperado de http://socnetv.org/

Kossinets, G. \& Watts, D. (2009). Origins of Homophily in an Evolving Social Network. American Journal of Sociology, 115 (2), 405-50.

Li, T., Ogihara, M. \& Tzanetakis, G. (2012). Music Data Mining. Boca Raton, Estados Unidos: CRC Press, Taylor \& Francis Group.

List, G. (1963). The Boundaries of Speech and Song. Ethnomusicology, 7, 1-16. 
Lomax, A. (1977). Appeal for Cultural Equity. Journal of Communication, 27, 125-138.

Luján, J. (2015). Etnomusicología de las músicas turbias: consumos culturales, raza y etnicidad. Esfera, 5 (1), 5-20.

Luján, J. (2016). Formas de producción sociocultural de la población afrojuvenil en la ciudad de Cali: El caso del rap caleño en la década del noventa (Tesis de maestría inédita). Universidad Distrital Francisco José de Caldas, Bogotá, Colombia.

Mitchell, T. (2001). Global Noise: Rap and Hip-hop Outside the USA. Middletown, Estados Unidos: Wesleyan University Press.

Ong, J. (2006). Oralidad y escritura tecnologías de la palabra. México D.F., México: Fondo de Cultura Económica.

Pardue, D. (2014). An Introduction to Hip Hop in Latin America and the Caribbean. En D. Hornand \& J. Shepherd (eds.). The Continuum Encyclopedia of Popular Music of the World Vol. 9, (pp. 356-357). Londres, Inglaterra: Continuum Books.

Reynoso, C. (2011). Redes sociales y complejidad: Modelos interdisciplinarios en la gestión sostenible de la sociedad y la cultura. Buenos Aires, Argentina: Sb.

Ritossa, D. \& Rickard, N. (2004). The relative utility of "pleasantness" and "liking" dimensions in predicting the emotions expressed by music. Psychology of Music, 32(1), 5-22.

Ruesch, J. \& Bateson, G. (1965). Comunicación: la matriz social de la psiquiatría. Buenos Aires, Argentina: Paidós.

Taleb, N. (2008). El cisne negro. El impacto de lo altamente improbable. Barcelona, España: Paidós.

Telles, E. (2014). Pigmentocracies: Ethnicity, Race, and Color in Latin America. Chapel Hill, Estados Unidos: The University of North Carolina Press.

Urrea, G. (2012). Transformaciones sociodemográficas y grupos socio-raciales en Cali a lo largo del siglo XX y comienzos del siglo XXI. En L. Cano (ed.). Historia de Cali, siglo XX Vol. I, (pp. 145-194). Cali, Colombia: Universidad del Valle.

Vélez, A. (2004). El Rap una oportunidad de vida social en construcción que llega con la experiencia (Trabajo de grado inédito). Universidad del Valle, Cali, Colombia.

Watts, D. (2004). The 'new' science of networks. Annual Review of Sociology, 30, 243-270.

Winkin, Y. (1976). La Nueva Comunicación. Barcelona: Kairós.

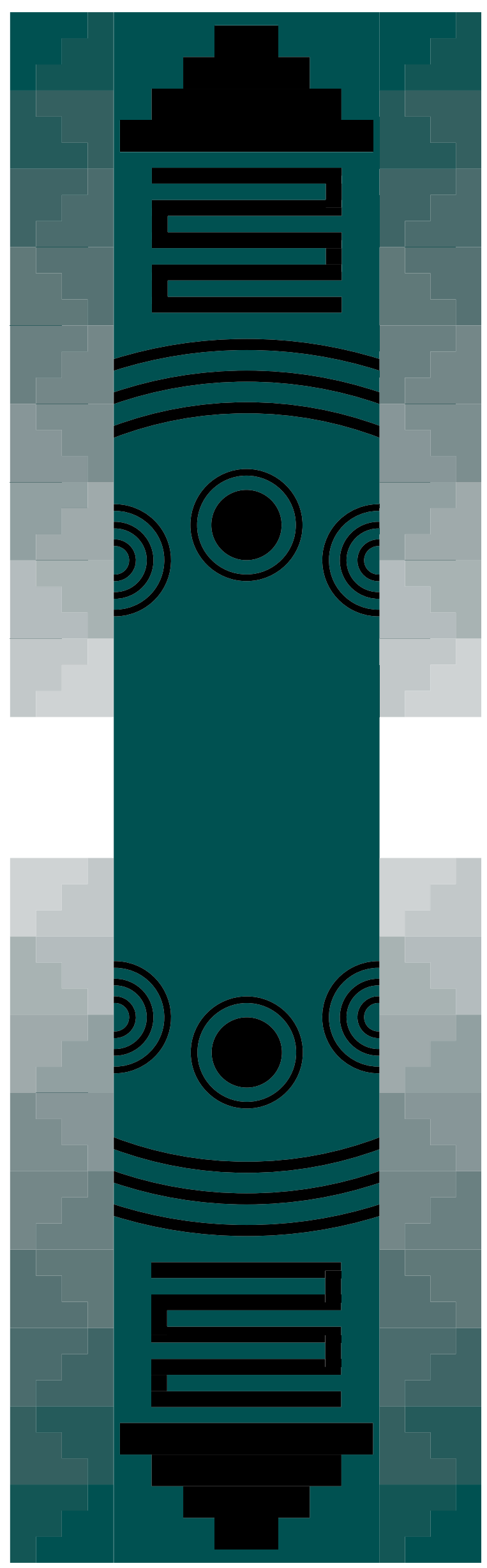

